\title{
A case report of medical management prior to surgical intervention in Molar pregnancy with SVT
}

\author{
${ }^{1}$ Nishanthi $V,{ }^{1}$ Aravinthan M, Sivansuthan $S^{1}$ \\ ${ }^{1}$ Teaching hospital Jaffna
}

\begin{abstract}
Gestational trophoblastic diseases comprise a spectrum of premalignant and malignant diseases, including hydatidiform mole (complete or partial), placental site trophoblastic tumor, choriocarcinoma and gestational trophoblastic neoplasia. Hydatidiform mole is the most common form of these (1). We report a case of 35-year-old women in her $5^{\text {th }}$ para with the POA of 12 weeks presented with the features of thyrotoxicosis complicated with multiple episodes of SVT found to have molar pregnancy. She underwent medical cardioversion and thyroid suppressive treatment in order to stabilize and prevent perioperative complications. She underwent surgery and complete recovery was noted. Even though the definitive treatment is surgical its essential to consider the medical management first to stabilize the patient prior to the surgery.
\end{abstract}

\section{Keywords}

Hydatiform mole, Supraventricular tachycardia (SVT), Thyrotoxicosis, period of amenorrhea (POA)

\section{Introduction}

Gestational trophoblastic disease (GTD) is a group of disorders that may develop following abnormal fertilization (2). The definitive treatment for GTD is surgical evacuation of the trophoblastic tissue (3). In cases of advanced GTD, patients must be evaluated for hyperthyroidism, and their hypermetabolic status and thyroid function must be optimized prior to surgery to prevent intraoperative complications (4).

\section{Case report}

A pregnant 35-year-old gravida 3 para 5 woman was admitted with the history of lower abdominal pain and mild PV bleeding at POA of 12 weeks. At presentation, she was normotensive, anxious, tachycardic and diaphoretic. ECG showed supraventricular tachycardia.

She was initially managed with adenosine followed by verapamil. The rhythm was reverted following verapmail. USS reveled that her uterus filled with heterogeneous echoic material suggestive of hydatiform mole. Her serum beta human chorionic gonadotropin ( $\beta$-HCG) level measured which was $>600,000 \mathrm{mIU} / \mathrm{L}$. Her serum free T4 level came as $6.09 \mathrm{mIU} / \mathrm{ml}(0.40-4.50)$ with $\mathrm{TSH}$ of $<0.015 \mathrm{mIU} / \mathrm{L}(0.4$ and 4.0$)$. She was managed as H.Mole related thyrotoxicosis and started on carbimazole and metoprolol. Urgent surgery was performed as she developed recurrent SVT during the stay and her thyroid status came back to normal during the follow up investigations.

\section{Discussion}

GTD presents with a combination of symptoms such as uterine enlargement greater than gestational dates, vaginal bleeding, hyperemesis, pregnancyinduced hypertension and hyperthyroidism. Clinical diagnosis is based on history, physical examination, pelvic ultrasound and serum $\beta$-HCG quantification $(>100,000 \mathrm{mIU} / \mathrm{mL})$. Treatment is urgent surgical evacuation and curettage, and pathologic examination of the curettage specimen confirms the diagnosis (5).

The patient's initial presentation can be explained by hyperthyroidism, secondary to high secretion of $\beta$-HCG from the trophoblastic tissue. There are 
well-documented evidences of molecular mimicry between the hCG and TSH subunits.

Dilation and curettage were considered for the patient; however, there were significant perioperative risks. Thus, it was decided that control of her hypermetabolic state and thyroid status prior to the surgical intervention. If the patient was clinically stable, dilation and curettage would have been performed urgently and without preceding medical management. There is inadequate evidence into a definitive length of time for medical management prior to surgical intervention to prevent thyrotoxicosis complications.

\section{Conclusion}

Hyperthyroidism is a serious complication associated with GTD. Due to the low prevalence of advanced GTD in developed countries, there is sparse literature discussing the optimal medical management of these patients. When a patient presents with advanced GTD, medical management of her hypertension and hyperthyroidism took priority over immediate surgical therapy in order to minimize the risk of anesthetic and surgical complications. But considering the cardiac arrhythmias which can be triggered by the thyroid status it's advisable to go for early surgical intervention to achieve full recovery.

\section{Reference}

1. Virmani S., Srinivas S.B., Bhat R., Rao R., Kudva R. Transient thyrotoxicosis in molar pregnancy. J. Clin. Diagn. Res. 2017;11(7):1-2.

2. Brown J., Naumann R.W., Seck1 M.J., Schink J. 15 years of progress in gestational trophoblastic disease: scoring standardization, and salvage. Gynecol Oncology. 2017;144:200207.

3. Samra T., Kaur R., Sharma N., Chaudhary L. Peri-operative concerns in a patient with thyroid storm secondary to molar pregnancy. Indian J Anesthesia. 2015;59(11):739-742.

4. Swaminathan S., James R.A., Chandran R., Joshi R. Anaesthetic implications of severe hyperthyroidism secondary to molar pregnancy: a case report and review of literature. Anesth. Essays Res. 2017;11(4):1115-1117.

5. Lurain J.R. Gestational trophoblastic disease I: epidemiology, pathology, clinical presentation and diagnosis of gestational trophoblastic disease, and management of hydatidiform mole. Am. J. Obstet. Gynecol. 2010;203(6):531-539. 